\title{
Premedication with intravenous steroids does not influence the incidence of infusion reactions following infliximab infusions in pediatric inflammatory bowel disease patients-a case-control study
}

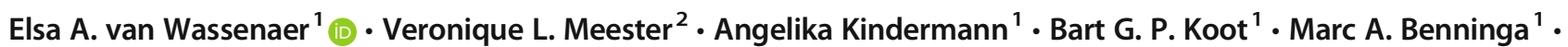 \\ Tim G. J. de Meij ${ }^{2}$
}

Received: 26 March 2019 / Accepted: 4 July 2019 / Published online: 22 July 2019

(C) The Author(s) 2019

\begin{abstract}
Purpose Infusion reactions (IR) are commonly described side effects of infliximab (IFX) infusions, often leading to discontinuation of IFX. This study aimed to investigate the influence of steroid premedication (PM) on incidence of IR in pediatric inflammatory bowel disease (PIBD) patients receiving IFX.

Methods A case-control study in two tertiary centers in Amsterdam, The Netherlands, including PIBD patients receiving IFX. $\mathrm{PM}$ with steroids was part of standard care in one center $(\mathrm{PM}+)$ but not in the other center (PM-). Acute IR were divided into mild/severe reactions and in grade 1/2/3/4 for detailed exploration. Differences between subgroups were assessed with the $T$ or chi-square test. Multivariate logistic regression was used to assess associations between PM and IR incidence, correcting for comedication usage.

Results We included 226 patients (91 PM+, 50\% male, mean age at onset of IBD 12.7 years), receiving 3433 infusions. There was no difference between the PM+ and PM- subgroups in incidence of IR (14.3\% vs. 17.0\% of patients, $p=0.58)$ and in percentage of infusions followed by IR (1.4\% in both subgroups). The OR of developing IR when using PM was 1.06 (95\% CI $0.49-2.27, p=0.89)$, and the OR of developing a grade 3 or 4 IR when using PM was $0.90(95 \%$ CI $0.24-3.39, p=0.88)$ when correcting for co-medication usage.

Conclusion The incidence of IR was low, and premedication with steroids did not decrease the incidence of IR in this cohort of PIBD patients receiving IFX. Our results indicate that PM with steroids is not indicated in PIBD to prevent IR.
\end{abstract}

Keywords Infliximab $\cdot$ Pediatric inflammatory bowel disease $\cdot$ Infusion reactions $\cdot$ Premedication

\section{Introduction}

Infliximab (IFX) is a monoclonal antibody with high specificity for TNF- $\alpha$, a pro-inflammatory cytokine playing an important role in the pathogenesis of inflammatory bowel disease (IBD) $[1,2]$. It has become a highly valuable therapy for IBD

Elsa A. van Wassenaer

e.a.vanwassenaer@amc.nl

Emma Children's Hospital, Amsterdam UMC, Pediatric Gastroenterology, University of Amsterdam, Meibergdreef 9, 1105 AZ Amsterdam, The Netherlands

2 Emma Children's Hospital, Amsterdam UMC, Pediatric Gastroenterology, Vrije Universiteit Amsterdam, Amsterdam, The Netherlands patients in adults as well as in children [3]. IFX is efficient both in inducing and in maintaining remission [4], although infusion reactions (IR) are relatively commonly observed side effects, especially in children (12.3-38.6\% of patients) [5-7]. No consensus exists on the exact definitions of IR [8], but most commonly described IR against IFX include pruritus, flushing, and dyspnea [2, 5]. The evidence on risk factors for development of IR is however limited [9, 10]. Premedication (PM), such as antihistamines and corticosteroids, has been considered to decrease the odds of developing IR [11]. However, PM may cause side effects as well and using PM leads to an elongated admission time. Additionally, in a large cohort study of adult IBD patients receiving IFX, administration of antihistamines as premedication seemed to increase the risk for IR $(2.49 \%$ vs. $1.32 \%$ if not used, $p<0.0001)$, while corticosteroids did not 
seem to significantly influence incidence rates of IR [5]. In children, several small retrospective studies, mostly without the inclusion of a control group, have not confirmed the beneficial effect of PM on the incidence of IR either [12, 13]. Overall, there is currently no consensus on the use of PM in pediatric IBD patients to IFX-related IR [14]. Particularly since the use of IFX is rapidly increasing worldwide, both in pediatric and in adult IBD patients, and because IR commonly lead to discontinuation of IFX, evidence on the effects of PM on IR is needed. This study aimed to investigate the influence of steroids as PM on the incidence of acute IFX-related IR in pediatric IBD patients.

\section{Methods}

\section{Data collection}

All patients diagnosed with IBD (Crohn's disease (CD), ulcerative colitis (UC), or indeterminate IBD (IBD-U)) in two tertiary care centers for pediatric IBD in Amsterdam (Amsterdam University Medical Centers, location AMC and VU medical center), under 18 years, and treated with IFX between 1998 and 2018, were included in this case-control study. In one of the centers, PM with steroids was routinely administered prior to all IFX infusions, while in the other center, administration of PM prior to IFX was not part of standard care.

The following parameters were collected from the medical charts: sex, age, phenotype, age at diagnosis, location of disease at diagnosis according to the Paris classification [15], age at first infliximab infusion, previous IBD-related medication, co-medication during infliximab treatment, cumulative number of infusions per patient, occurrence and type of IR, number of infusions prior to occurrence of an IR, ATI levels, type of infliximab (Inflectra ${ }^{\circledR} /$ Remsima $^{\circledR} /$ Remicade $^{\circledR}$ ), and medication administered upon occurrence of IR.

\section{Administration of infliximab}

At the initiation of the IFX treatment, all patients were administered $5 \mathrm{mg} / \mathrm{kg}$ body weight infliximab with an infusion time of $2 \mathrm{~h}$. The infusions were scheduled on weeks 0,2 , and 6 , followed by intervals of 8 weeks. Adjustments on interval and dosage of infliximab were made by the clinician, based on clinical disease activity, IFX levels, and presence of antibodies. All infusions were administered and observed by trained nurses at a specialized day-care center, and adverse events including IR were documented. All IBD patients on IFX aged 5 years and older in the VU medical center routinely received PM with $100 \mathrm{mg}$ intravenous hydrocortisone, $30 \mathrm{~min}$ before administration of infliximab, with a 10-min infusion time. Patients younger than 5 years received $50 \mathrm{mg}$ intravenous hydrocortisone. Patients who were treated with steroids as remission-induction therapy for IBD or for concomitant auto-immune hepatitis while on infliximab therapy, did not receive additional hydrocortisone. This infusion protocol was used during the whole study period.

\section{Definition of infusion reaction}

IR against IFX were defined in two ways. First, the reactions were divided into mild or severe (Table 1) [16]. A reaction was considered mild when it was self-limiting or when it disappeared when infusion was slowed down or paused and subsequently continued, and severe when discontinuation of the infusion was required. However in several cases, the infusion could be continued in a later stage with extra precaution. To avoid any bias by categorizing IR as severe, while in fact the infusion was continued later on, a more accurate definition adapted from the Common Terminology Criteria for Adverse Events (CTCAE) from the National Cancer Institute USA (NCI) [17] was used (Table 1).

\section{Statistical analysis}

In case of normally distributed data, means and standard deviations were calculated, and in case of non-normally distributed data, medians and interquartile ranges were calculated. Differences between demographic variables in the PM and non-PM groups were compared using the unpaired $T$ test in case of normally distributed variables, using the MannWhitney $U$ test in case of non-normally distributed variables, and using the chi-square or Fisher's exact test in case of categorical variables. To assess the influence of PM on IR, we used multivariate logistic regression. We anticipated the presence of ATI to be the largest confounder in this study, but as data on ATI levels were missing in nearly half of the cases, we only corrected for usage of co-medication. Co-medication was defined as using an immunomodulator (any thiopurine or methotrexate) started within 1 month after the start of IFX therapy and continued for at least 6 months. All statistical analyses were performed with SPSS Statistics 25. $p$ values of $<0.05$ were considered statistically significant.

\section{Results}

\section{Patient characteristics}

A total of 226 patients, receiving combined 3433 single infusions, were included in this study. Patient characteristics are displayed in Table 2. The mean number of infusions per patient was 15 (SD 12.3, range 1-75). There were significant differences between the PM and non-PM groups regarding phenotype, disease extension and severity, and previous usage 
Table 1 Classification of infusion reactions

\begin{tabular}{ll}
\hline Mild & Self-limiting or disappears when infusion is slowed down or paused \\
Severe & Discontinuation of the infusion is required \\
Grade 1 & No interventions necessary, only observation \\
Grade 2 & Interruption of infusion and/or oral medication required \\
Grade 3 & Intravenous medication is necessary \\
Grade 4 & Infusion is required to stop
\end{tabular}

of immunomodulation. Also, patients in the non-PM group had received significantly more infusions.

\section{Infusion reactions}

In the PM group, a total of 15 IR (1.4\% of all infusions) occurred in $13(14.3 \%)$ patients, compared with a total of 34 IR (1.4\% of all infusions) in $23(17.0 \%)$ patients in the nonPM group (Figs. 1 and 2a, b). Three severe IR were reported, all in the PM group, and four grade 4 IR were reported, of which three in the PM group. Severe reactions included dyspnea and angioedema. Other reported IR were headache ( $n=$ 7); nausea and/or vomiting $(n=6)$; rash $(n=6)$; chest pain $(n=4)$; dizziness $(n=2)$; and chills, pruritus, edema, and temperature rise $(n=1)$. The number and severity of IR per person are described in Figs. 1 and 2. The first infusion reaction $(n=$ 36 ) occurred averagely at the 6th infusion (SD 5.72, range 1$24)$. The second infusion reaction $(n=11)$ occurred averagely at the 7th infusion (SD 5.52, range 2-18), and the third $(n=2)$ at the 6th infusion (SD 3.54, range 3-8).

In the non-PM group, 9 (6.7\%) patients eventually did receive premedication because of previous IR. Four of those did not experience another infusion reaction anymore and three did. In two patients, documentation was too limited to tell.

Table 2 Baseline characteristics

\section{Influence of premedication}

The number of patients developing any infusion reaction did not differ statistically significant between the PM and non-PM groups ( $13 / 91$ vs. $23 / 135, p=0.58$ ) (see Fig. $2 a$ ), and neither did the number of patients developing a grade $3 / 4$ infusion reaction (4/91 vs. 6/136, $p=0.99$ ) (see Fig. $2 c$ ). There also was no difference in the relative amount of IR between both groups (median 0 (IQR $0-0$ ) vs. 0 (IQR $0-0), p=0.75$ ). The odds ratio (OR) of developing any infusion reaction when using PM, corrected for using co-medication, was 1.06 (95\% CI 0.49-2.27, $p=0.89$ ). The OR of developing a grade 3 or 4 IR when using PM was 0.90 (95\% CI 0.24-3.39, $p=0.88$ ), also corrected for using co-medication.

\section{Risk factors for IR}

ATI levels against IFX were measured in 104 patients (46\%) and were present in 24 patients $(11 \%)(10(11.0 \%)$ in the PM group and $14(10.4 \%)$ in the non-PM group). Of these 24, six developed IR. Due to the substantial amount of missing data, we could not perform an analysis on the influence of ATI on IR. The number of patients using co-medication in both

\begin{tabular}{llll}
\hline & $\mathrm{PM}+(n=91)$ & $\mathrm{PM}-(n=135)$ & $p$ value \\
\hline Age at onset (mean, SD) & $12.5(3.3)$ & $12.8(3.1)$ & 0.55 \\
Males (\%) & $45(49.5)$ & $70(51.9)$ & \\
Crohn's disease (\%) & $61(67.0)$ & $114(84.4)$ & 0.007 \\
Ulcerative colitis (\%) & $24(26.4)$ & $15(11.1)$ & 0.007 \\
IBD-U (\%) & $6(6.6)$ & $6(4.4)$ & 0.007 \\
Previous IBD medication (\%) & & & \\
Corticosteroids & $71(78)$ & $96(69.9)$ & 0.23 \\
Immunomodulator & $68(74.4)$ & $122(89.7)$ & 0.005 \\
Co-medication & $74(81.3)$ & $88(65.2)$ & 0.008 \\
$\quad$ Azathioprine & $58(63.7)$ & $76(56.3)$ & 0.05 \\
Mercaptopurine & $2(2.2)$ & $3(2.2)$ & 0.05 \\
Thioguanine & $2(2.2)$ & - & - \\
$\quad$ Methotrexate & $12(12.2)$ & $9(6.7)$ & 0.05 \\
$N$ of infusions per patient (mean, SD) & $11.9(9.4)$ & $17.4(13.5)$ & 0.001 \\
\hline
\end{tabular}


Fig. 1 Flowchart of number and severity of infusion reactions (IR) in the premedication $(\mathrm{PM}+)$ and non-premedication (PM-) groups. *One missing value for IR severity

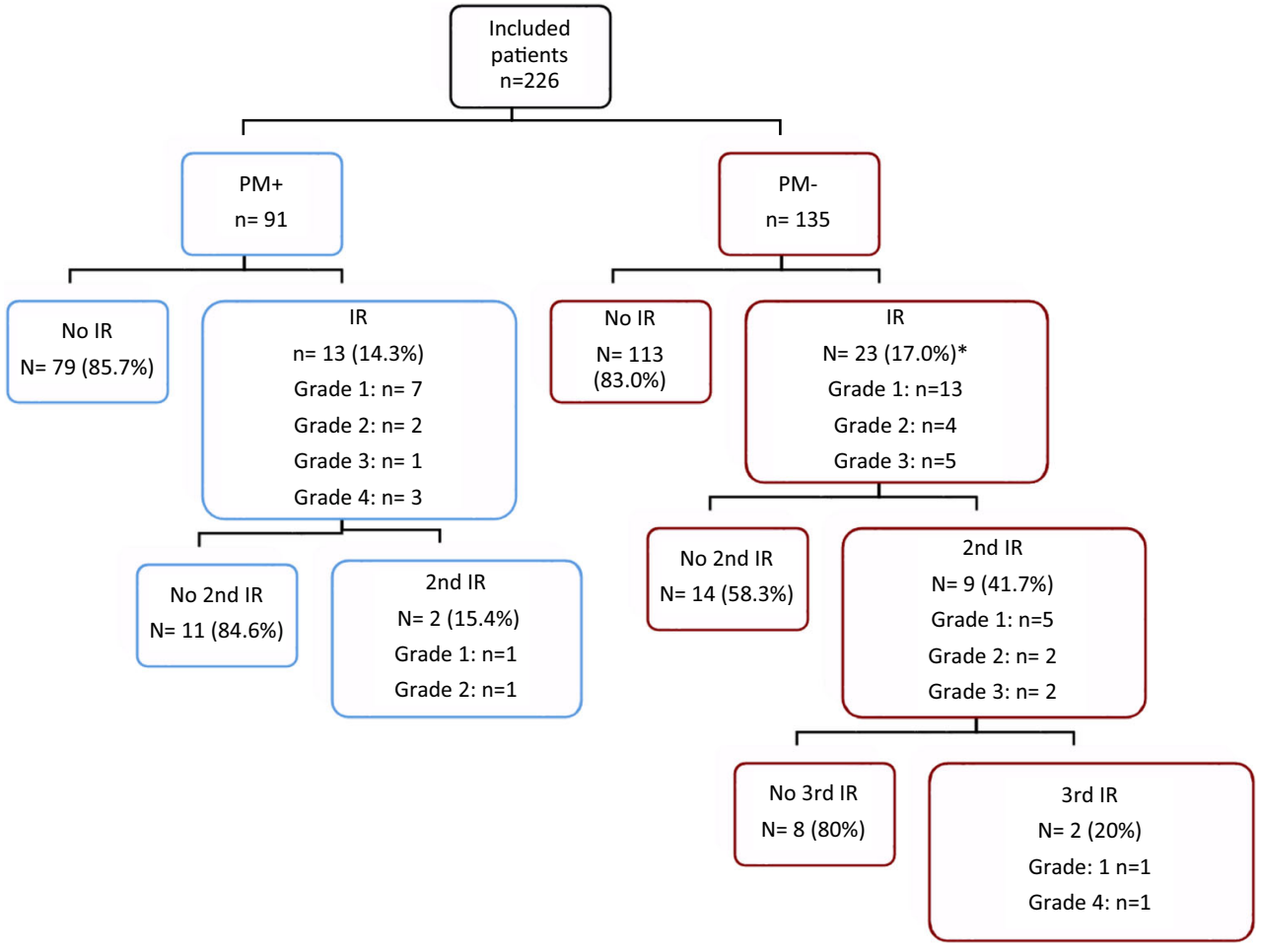

groups is depicted in Table 2. Using co-medication did not influence the risk of developing IR $(p=0.39)$.

The effect of drug holidays on IR incidence could not be assessed reliably, as this was only reported in 3 patient charts. Two of these patients did develop an infusion reaction and the other one did not.

\section{Discussion}

In this cohort of pediatric IBD patients receiving IFX infusions, premedication (PM) with steroids was not associated with a decreased incidence of IR; the number of patients developing IR was comparable in both groups.
Fig. 2 a Percentage of patients developing first, second, or third infusion reaction (IR) in the premedication $(\mathrm{PM}+)$ and nonpremedication (PM-) groups. b Percentage of infusions resulting in first, second, or third IR in PM+ and PM- groups. c Percentage of patients developing grade 3 or 4 IR in $\mathrm{PM}+$ and $\mathrm{PM}-$ groups
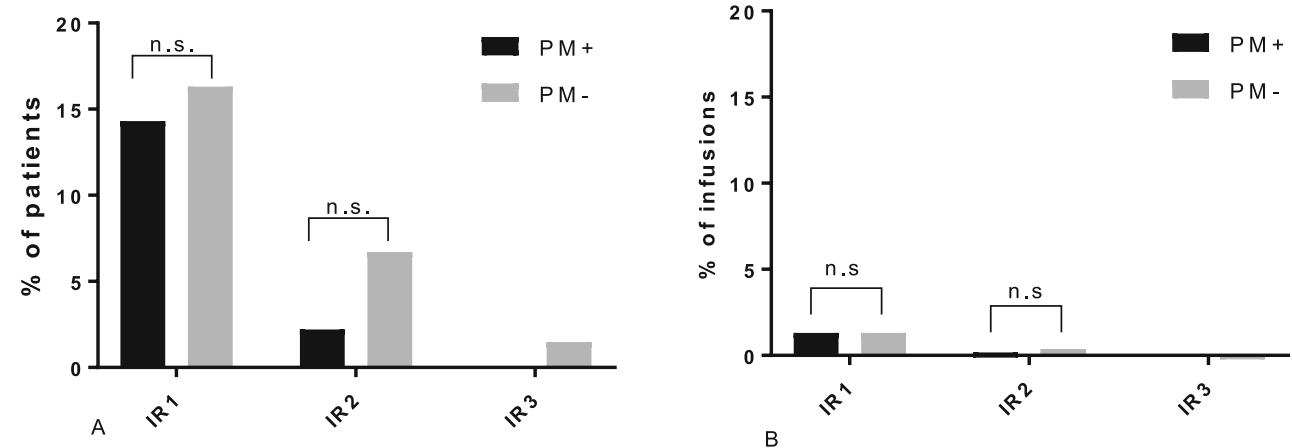
The usage of PM prior to IFX administration has been subject of debate both in adult and pediatric IBD populations $[11,14]$. In the pediatric setting, only few studies have addressed this issue, of which most studies were underpowered. Additionally, most of these studies did not distinguish between different types of PM, so no firm conclusions could be drawn $[12,13,18]$. A recent retrospective study including a larger cohort of pediatric IBD patients also assessed the influence of PM with corticosteroids, antihistamines, and acetaminophen on risk for IR [19]. In that study, administration of corticosteroids was associated with a higher risk for IR (RR 8.42, 95\% CI 4.6-15.3) while antihistamines seemed to have a protective effect on the development of IR (RR $0.26,95 \%$ CI $0.14-0.64)$. However, the number of patients receiving corticosteroids as PM was low ( $n=30,402$ infusions), and the usage of PM was not standardized as in our cohort, which might have led to selection bias. Our findings are in line with those of a large prospective cohort study in adult IBD patients, including 1632 patients on IFX. In $22.5 \%$ of the infusions, steroids were given as PM, but this strategy did not seem to result in a decreased incidence of IR [5].

Because of our sample size and the limited amount of data on ATI that could be derived from the charts, we could not assess any risk factors for developing IR such as ATI. However, in the study of Miele et al. [10], a significant difference was found between the number of IR between pediatric IBD patients with and without ATI (13.8\% of 94 infusions vs. $3.6 \%$ of 140 infusions, respectively; $p<0.01$ ). The same study and others also identified the usage of concomitant immunomodulation as a negative risk factor for development of ATI [20]. To minimize bias due to confounding by unknown ATI levels in our population, we used concomitant immunomodulatory therapy as covariate, as this significantly reduces ATI-forming [21]. Other studies also found an association between drug holidays and IR $[9,16]$, but this could not be confirmed in our study.

This study has several limitations. Due to the retrospective design, not all baseline characteristics could be collected, and reporting of IR by the nurse was not done on a standardized matter. This may have led to an underestimation of IR. However, it is worth noting that severe reactions are always reported by the nurses in both hospitals, while the pharmacy reports have been checked for the issuing of any emergency medication. For this reason, we also assessed the effect of PM on solely grade 3 and 4 IR. Categorizing IR may be challenging in retrospective studies, due to the lack of standardization of reporting of any IR among nurses in both hospitals. However, categorizing IR is of great importance, because reported incidence of IR in different studies highly depends on the used definitions for IR [8]. For this reason, we chose to include only acute reactions, as delayed reactions are not well reported in most cases. Subsequently, we decided to subdivide reported IR into different categories, as IR which require medication or even cessation of the infusion are of greater clinical importance than, for example, a self-limiting, mild headache. Another limitation is the difference in baseline characteristics between both groups: there were significant differences in disease phenotype for example. However, this variable has not been reported to contribute as a risk factor for development of IR, so we believe the influence of this difference is of limited importance. More importantly, there was a significant difference in usage of concomitant immunomodulatory therapy. But as mentioned above, we minimized subsequent bias by entering this as covariate in our logistic regression model.

Another limitation of this study is the sample size. We included every pediatric IBD patient that received one or more IFX infusion in the two participating tertiary care centers, which led to inclusion of as much as 226 subjects and 3433 infusions. Although this is a relatively large group considering the prevalence of IBD among children in Europe, this study is still underpowered to detect very small differences in incidence of IR. To reach an appropriate $(80 \%)$ power, a future, preferably prospective, controlled study would need approximately 2000 participants per treatment group to detect the same difference we found. It could be questioned if this is feasible and, more importantly, whether this is clinically relevant, taking into account the low incidence of significant IR, as observed in both subgroups from the present study: only eight grade 3 and four grade 4 reactions out of the 3433 infusions. However, it would be interesting to investigate the effect of other types of PM, like antihistamines, in high-risk patients, such as patients with a history of an infusion reaction or patients with elevated ATI.

The present study is the largest case-control study in which PM with steroids was routinely given in one of the groups and not in the other groups of pediatric IBD patients, which minimized the risk of selection bias. Even though the two groups were collected from two different hospitals, the patient populations had similar demographic characteristics, as both hospitals are located in one city and cover the same area and use similar protocols - besides the administration of PM.

In conclusion, in our cohort consisting of 226 IBD children on IFX, with 3433 single IFX infusions, the incidence of IR was relatively low. Administration of PM with corticosteroids did not influence IR rates. Therefore, and also based on previous study outcomes, we advise not to routinely prescribe steroids as premedication prior to IFX in pediatric IBD patients.

Author contributions EAvW, VLM, and TGJdM performed the research. EAvW analyzed the data and wrote the paper. VLM and EAvW collected the data. TGJdM designed the research study. AK, BGPK, and MAB contributed to the design of the study and helped writing the paper.

\section{Compliance with ethical standards}

Conflict of interest The authors declare that they have no conflicts of interest. 
Ethical approval This study was performed in compliance with the Declaration of Helsinki.

Informed consent Not applicable, as this was a retrospective chart review.

Open Access This article is distributed under the terms of the Creative Commons Attribution 4.0 International License (http:// creativecommons.org/licenses/by/4.0/), which permits unrestricted use, distribution, and reproduction in any medium, provided you give appropriate credit to the original author(s) and the source, provide a link to the Creative Commons license, and indicate if changes were made.

\section{References}

1. Scallon BJ, Moore MA, Trinh $\mathrm{H}$ et al (1995) Chimeric anti-TNFalpha monoclonal antibody cA2 binds recombinant transmembrane TNF-alpha and activates immune effector functions. Cytokine 7: 251-259. https://doi.org/10.1006/cyto.1995.0029

2. Parashette KR, Makam RC, Cuffari C (2010) Infliximab therapy in pediatric Crohn's disease: a review. Clin Exp Gastroenterol 3:57-63

3. Ruemmele FM, Veres G, Kolho KL, Griffiths A, Levine A, Escher JC, Amil Dias J, Barabino A, Braegger CP, Bronsky J, Buderus S, Martín-de-Carpi J, de Ridder L, Fagerberg UL, Hugot JP, Kierkus J, Kolacek S, Koletzko S, Lionetti P, Miele E, Navas López VM, Paerregaard A, Russell RK, Serban DE, Shaoul R, van Rheenen P, Veereman G, Weiss B, Wilson D, Dignass A, Eliakim A, Winter H, Turner D (2014) Consensus guidelines of ECCO/ESPGHAN on the medical management of pediatric Crohn's disease. J Crohn's Colitis 8:1179-1207. https://doi.org/10.1016/j.crohns.2014.04.005

4. Corica D, Romano C (2017) Biological therapy in pediatric inflammatory bowel disease a systematic review. J Clin Gastroenterol 51: 100-110. https://doi.org/10.1097/MCG.0000000000000696

5. Choquette D, Faraawi R, Chow A, Rodrigues J, Bensen WJ, Nantel F (2015) Incidence and management of infusion reactions to infliximab in a prospective real-world community registry. J Rheumatol 42:1105-1111. https://doi.org/10.3899/jrheum. 140538

6. Kolho KL, Ruuska T, Savilahti E (2007) Severe adverse reactions to infliximab therapy are common in young children with inflammatory bowel disease. Acta Paediatr Int J Paediatr 96:128-130. https://doi.org/10.1111/j.1651-2227.2007.00042.x

7. Crandall WV, Mackner LM (2003) Infusion reactions to infliximab in children and adolescents: frequency, outcome and a predictive model. Aliment Pharmacol Ther 17:75-84. https://doi.org/10.1046/ j.1365-2036.2003.01411.x

8. Vande Casteele N, Breynaert C, Vermeire S, Rutgeerts PJ, Gils A, van Assche G (2011) Incidence of acute severe infusion reactions to infliximab depends on definition used rather than assay. Aliment Pharmacol Ther 34:401-403. https://doi.org/10.1111/j.1365-2036. 2011.04726.x

9. Duron C, Goutte M, Pereira B, Bommelaer G, Buisson A (2015) Factors influencing acute infusion reactions in inflammatory bowel disease patients treated with infliximab in the era of scheduled maintenance therapy. Eur J Gastroenterol Hepatol 27:705-711. https://doi.org/10.1097/MEG.0000000000000354
10. Miele E, Markowitz JE, Mamula P, Baldassano RN (2004) Human antichimeric antibody in children and young adults with inflammatory bowel disease receiving infliximab. J Pediatr Gastroenterol Nutr 38:502-508. https://doi.org/10.1097/00005176-20040500000008

11. Lichtenstein L, Ron Y, Kivity S, Ben-Horin S, Israeli E, Fraser GM, Dotan I, Chowers Y, Confino-Cohen R, Weiss B (2015) Infliximabrelated infusion reactions: systematic review. J Crohn's Colitis 9: 806-815. https://doi.org/10.1093/ecco-jcc/jjv096

12. Jacobstein DA, Markowitz JE, Kirschner BS, Ferry G, Cohen SA, Gold BDW, HS HMBBR (2005) Premedication and infusion reactions with infliximab: results from a pediatric inflammatory bowel disease consortium. Inflamm Bowel Dis 11:442-446

13. Hutsell SQ, Wu M, Park KT (2017) Frequency of severe infusion reactions associated with outpatient infusion of infliximab without premedications. J Pediatr Gastroenterol Nutr 65:430-431. https:// doi.org/10.1097/MPG.0000000000001535

14. Adler J, Sandberg KC, Shpeen BH, Eder SJ, Dhanani M, Clark SJ, Freed GL (2013) Variation in infliximab administration practices in the treatment of pediatric inflammatory bowel disease. J Pediatr Gastroenterol Nutr 57:35-38. https://doi.org/10.1097/MPG. 0b013e31828flea2

15. Levine A, Koletzko S, Turner D, Escher JC, Cucchiara S, de Ridder L, Kolho KL, Veres G, Russell RK, Paerregaard A, Buderus S, Greer MLC, Dias JA, Veereman-Wauters G, Lionetti P, Sladek M, Carpi JM, Staiano A, Ruemmele FM, Wilson DC (2014) ESPGHAN revised Porto criteria for the diagnosis of inflammatory bowel disease in children and adolescents. J Pediatr Gastroenterol Nutr 58:795-806. https://doi.org/10.1097/MPG. 0000000000000239

16. Steenholdt C, Svenson M, Bendtzen K, Thomsen OØ, Brynskov J, Ainsworth MA (2011) Severe infusion reactions to infliximab: aetiology, immunogenicity and risk factors in patients with inflammatory bowel disease. Aliment Pharmacol Ther 34:51-58. https:// doi.org/10.1111/j.1365-2036.2011.04682.x

17. U.S. Department of Health and Human Services (2017) Common terminology criteria for adverse events. In: Publish. http://ctep. cancer.gov/protocolDevelopment/electronic_applications/docs/ ctcaev3.pdf. Accessed 30 Jul 2018

18. Lahdenne P, Wikström AM, Aalto K, Kolho KL (2010) Prevention of acute adverse events related to infliximab infusions in pediatric patients. Arthritis Care Res (Hoboken) 62:785-790. https://doi.org/ 10.1002/acr.20246

19. El-Matary W, Dykes DMH, Bauman L et al (2017) Rapid infliximab infusion in children with inflammatory bowel disease: a multicenter North American experience. Inflamm Bowel Dis 23: 2104-2108. https://doi.org/10.1097/MIB.0000000000001259

20. Bots S, Gecse K, Barclay M, D'Haens G (2018) Combination immunosuppression in IBD. Inflamm Bowel Dis 24:539-545. https:// doi.org/10.1093/ibd/izx065

21. Colombel JF, Sandborn WJ, Reinisch W, Mantzaris GJ, Kornbluth A, Rachmilewitz D, Lichtiger S, D'Haens G, Diamond RH, Broussard DL, Tang KL, van der Woude C, Rutgeerts P, SONIC Study Group (2010) Infliximab, azathioprine, or combination therapy for Crohn's disease. N Engl J Med 362:1383-1395. https://doi. org/10.1056/NEJMoa0904492

Publisher's note Springer Nature remains neutral with regard to jurisdictional claims in published maps and institutional affiliations. 